\title{
Experimental Research of Wall Pressure Distribution and Effect of Micro Jet at Mach
}

1.5

\author{
Muhammed Hanafi Azami, Mohammed Faheem, Abdul Aabid, Imran Mokashi, S. A. Khan
}

\begin{abstract}
In this paper, a study on the effect of the control on the wall pressure as well as the quality of the flow when tiny jets were employed. The small jet aimed to regulate the base pressure at the base region of the suddenly expanded duct and wall pressure distribution is carried out experimentally. The convergent-divergent (CD) nozzle with a suddenly expanded duct was designed to observe the wall pressure distribution with and without control using small jets. In order to obtain the results with the effect of controlled four tiny jets of $1 \mathrm{~mm}$ diameter located at a ninety-degree interval along a pitch circle diameter (PCD) of 1.3 times the CD nozzle exit diameter in the base, region was employed as active controls. The Mach numbers of the rapidly expanded are 1.5. The jets were expanded quickly into an axis-symmetry duct with an area ratio of 4.84. The length-todiameter $(L / D)$ ratio of the rapid expansion duct was diverse from 10 to 1 . There is no adverse effect due to the presence of the tiny jets on the flow field as well as the quality of the flow in the duct.
\end{abstract}

Keywords-CD Nozzle, Micro jet, Wall Pressure, and Mach number.

\section{INTRODUCTION}

Sudden expansion is a widespread phenomenon that occurs in many engineering problems. The pressure at the blunt base of a projectile, the fuselage of the aircraft, base of the shell fired from artillery, unguided rockets, and the missiles. The pressure in the wake region will be subatmospheric which will result in negative base pressure coefficient leading to a considerable amount of drag which two-thirds of the total drag of the aerodynamic object. There are two methods to regulate the flow in the wake region. The first is the active control and the second one is the passive control. In the case of passive regulations, we make the geometrical change in the shape of the suddenly expanded duct so that the flow field is modified and hence results in an increase/decrease in the base pressure. The application of the control mechanism also depends on the requirements for a given situation/mission requirement. If the mission requirement is to decrease the base pressure as low as possible as in the case of the combustion chamber for efficient combustion and the efficiency of the combustor. However, in the case of external aerodynamics applications, the requirement is to increase the base pressure almost equal

Revised Manuscript Received on July 10, 2019.

Muhammed Hanafi Azami, Dept. of Mechanical Engineering, Faculty of Engineering, International Islamic University, Kuala Lumpur, Malaysia, (hanafiazami@iium.edu.my)

Mohammed Faheem, Dept. of Mechanical Engineering, Faculty of Engineering, International Islamic University, Kuala Lumpur, Malaysia.

Abdul Aabid, Dept. of Mechanical Engineering, Faculty of Engineering, International Islamic University, Kuala Lumpur, Malaysia.

Imran Mokashi, Dept. of Mechanical Engineering, Faculty of Engineering, International Islamic University, Kuala Lumpur, Malaysia.

S. A. Khan, Dept. of Mechanical Engineering, Faculty of Engineering, International Islamic University, Kuala Lumpur, Malaysia. to the ambient pressure, or in some cases, where we want to increase the base pressure manifolds in some special cases of external ballistics problems. For passive control, people have tried to control the flow with ribs, boattail, cavities, ventilated cavity, step body, splitter plate, and vortex locking mechanism to enhance the base pressure. Vented cavities were found to be very effective, without paying the penalty in terms of increment in the mass of the weapon system. In case of using the boattail at the rear of the base of the shells, due to the decrease in the base diameter, there will be a decrease in the base pressure as the base pressure coefficient is multiplied by the ratio of the base diameter to the maximum diameter square. But while using the boattail one should always keep in mind that due to the boattail angle should be small. If the boattail angle is more, then, in this case, the large angle will lead to flow separation at the base, which is not a desirable thing to happen. At the same time if the boattail angle is minimal, then boattail length becomes large which will result in addition skin friction drag coefficient. Hence while using the passive control one has to optimize the geometrical parameters. In the For dynamic control, base-bleed as a control mechanism has been very effective when the air is injected in the wake region, and that results in raising the base suction at the base. In the area of projectiles/rockets during high-speed flows; the expanded flow has many applications in the exterior ballistics. The convergent-divergent (CD) nozzle flow in the base region of an expanded tube with a cylindrical shape has low-pressure values. [1], [2], [3], [4]. During these circumstances in maximum cases, the base pressure is lower than the atmospheric pressure. As a result, the base drag is considerable. The shear layer when approaches out of the CD nozzle and is reattached with the duct and this point is referred to as reattachment point as shown in figure 1[5]. The reattachment

When the flow is exposed on the suddenly expanded area, which exists to the shear layer, the length to diameter ratio (L/D) of suddenly expanded duct, the expansion level, Nozzle pressure ratio (NPR) and Mach number all affect the base pressure. The flow recirculation arises due to suction created in the base region and because of the existence of a dominant vortex in the base of the tube[6], [7]. Many of the researchers have studied and solved this type of problem using computational fluid dynamics (CFD) method [8], [9], [2] and for supersonic flows over the wedge[10]. However, the study has also been performed in a CD nozzle for

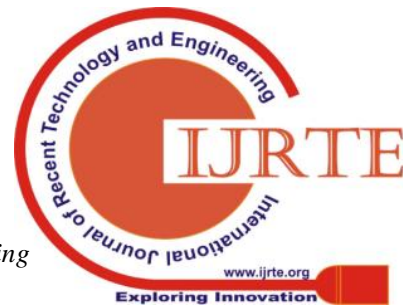


suddenly expanded duct with the effect of Mach number, $\mathrm{L} / \mathrm{D}$, area ratio and total thrust force of the duct.

\section{EXPERIMENTAL WORK}

The experimental set is replicated to indicate that the projectile base pressures are related to the upstream boundary layer type, thickness, and the location of the transition point.[11] The thickness of the boundary layer just upstream of the corner determines the base pressure for projectiles and slenderness ratio that is the L/D ratio[12]. The main structures of the suddenly expanded flow field are shown in Fig. 1 showing the reattachment point, expansion waves, and recirculation zone. Fig. 2 demonstrates the setup used for the experiments in the present study as discussed in Ref. [13]. The same concept is used to perform the experimental investigation with the application of four tiny jets at the base as shown in Figure 2. Figure 2 demonstrates the setup used for the experiment in the present study which is also discussed in [14].

The experiments were conducted at high-speed aerodynamics laboratory with an open jet facility.

The wind tunnel tests were done with an experimental model consisting of $\mathrm{CD}$ nozzles, pipelines, pressure transducers, and the settling chamber. In order to expand the gas through the nozzle and the duct, it is first allowed to go through regulating valves. The flow leaving the duct is exposed to air of ambient atmospheric pressure. Fig. 2 depicts the experimental setup with the nozzle and duct where the flow expands. At the outlet boundary of the nozzle, eight holes of $1 \mathrm{~mm}$ diameter each are drilled [15], [16]. Control of base pressure is achieved by injecting the air through the control holes. Wall pressure tapes were positioned along the duct to measure the wall pressure as well as the quality of the flow in the duct[17]. The pressure range is $0-300$ psi of the transducer employed, and it has 16 channels. The sampling rate of the pressure transducer is two hundred and fifty samples per second, and then the reading is displayed on the monitor and recorded on the monitor. The wall pressure was recorded using mercury manometer.

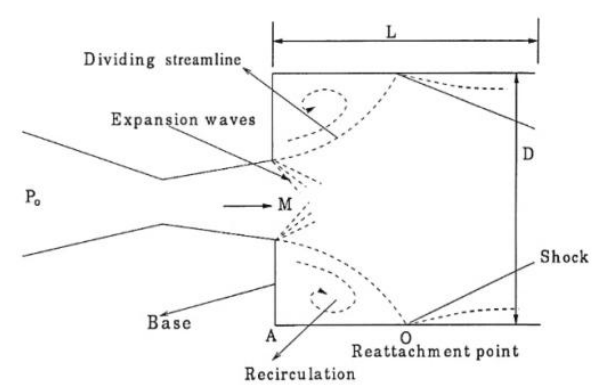

Figure 1: Sudden Expansion Flow Field

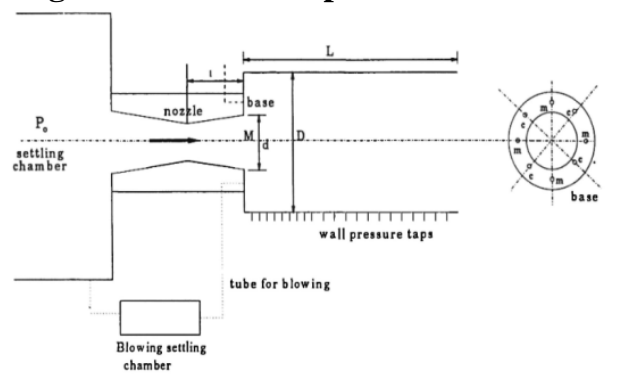

Figure 2: Experimental Setup.

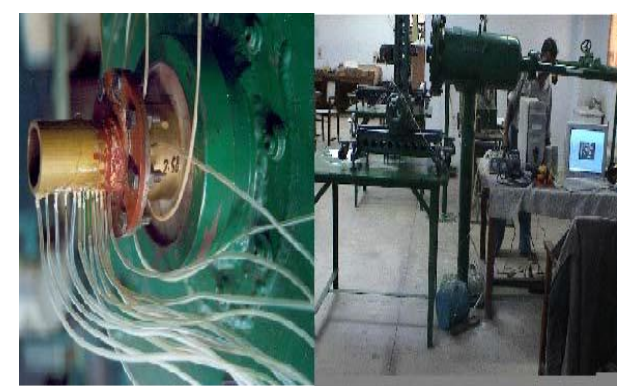

Figure 3 A View of Suddenly Expanded Duct with Pressure Tapings and setup location

\section{RESULTS AND DISCUSSION}

The wall pressure results for area ratio 4.84 and Mach 1.5 are presented in Figs. 4, for all the L/Ds of the present tests. The area ratio 4.84 is the case of slightly increased relief for the expanding flow in comparison to the lower area ratios. Since the location of the microjets as the control mechanism was fixed hence due to the increase in the area ratio, the microjets have further shifted away from the base and tend to go near the main jet, and the microjets do not adversely influence the wall pressure field [18].

Wall pressure results for Mach 1.5 are shown in Figs. 4. It is seen from these results that, for $L / D=10$ and $M=1.5$ the control influence is minimal (as in the case of Figs. 4 (a) and it does not augment the wall pressure adversely. There is a marginal change in the peak values of the wall pressure for the higher NPR. The wall pressure results for $\mathrm{L} / \mathrm{D}=10$ show that the NPR of the tests are such that the jets are over expanded, correctly expanded, and under expanded. When the jet are over expanded the initial wall pressure values are quite high due to the presence of the oblique shock wave at the exit of the nozzle. When the tested NPR is equal to the one required for correct expansion then the flow field in the duct possess minimal oscillation. When the jets are under expanded in that case the pressure at the nozzle exit will be more than the ambient atmospheric pressure. When the jet is exiting from the nozzle, at the exit of the nozzle an expansion fan will be sitting there through which the will continue to expand till the pressure is reduced considerably to become equal to the atmospheric pressure. When the supersonic jet is exiting from the nozzle it will be get divided in two main regions, one is the main jet and the other is recirculation zone separated by the dividing streamline. This separated flow will get attached again with the duct wall. From this reattachment point again there will be a shock wave as well as the the growth of the boundary layer. Further, it is seen that for NPR 3, 5, and 7 the fluctuations in the wall pressure are at the minimum level, and they are limited within the $10 \%$ distance from the exit of the duct diameter. Whereas for all the NPR the static wall pressure flow field by itself is oscillatory in nature due the flow being over, under, and ideally expanded, and hence, due to the small increase in the relief available to the flow, the flow will not be oscillatory in nature as the shear layer exiting from the nozzle gets increased area to expand and the control effectiveness is only marginal [19].

Blue Eyes Intelligence Engineering \& Sciences Publication

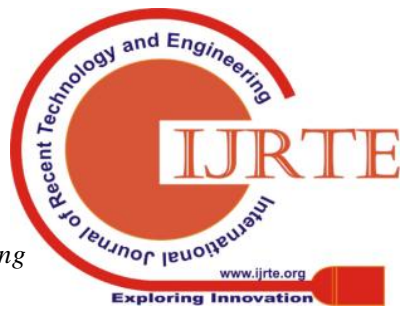


Fig. 4 (b) presents the similar wall pressure results for $\mathrm{L} / \mathrm{D}=8$ as was observed in the previous figure with the exception that the oscillations have reduced considerably as compared to $\mathrm{L} / \mathrm{D}=10$. Figs. 4 ((c) to (d)) represent the wall pressure results for $\mathrm{L} / \mathrm{D}=6$ and 5 with the exception that due reduction in the $\mathrm{L} / \mathrm{D}$ ratio there is some influence of back pressure and the peak pressure values are higher than that those were for higher $\mathrm{L} / \mathrm{D}$ ratios namely at $\mathrm{L} / \mathrm{D}=5$. It is also seen that the maximum increase in the wall pressure is twenty pressure more than the ambient atmospheric pressure. The magnitude of the wall pressure is low at lower NPR. With the increase in the NPR, the wall pressure also attains higher values of the wall pressure. Moreover, also the oscillatory nature of the wall has reduced considerably (L/D $=10$ and 8 ) respectively due to the increased area ratio and hence the relief available to the flow at the exit of the nozzle. It is also seen that the flow field once again has smoothened in the duct and the wall pressure values with and without control are the same. This trend continues till $\mathrm{L} / \mathrm{D}=4,3$ and 2 (Figs. 4 ((e) to $(\mathrm{g})$ ), then later for lowest $\mathrm{L} / \mathrm{D}$ like $\mathrm{L} / \mathrm{D}=1$, it is evident that this length is not enough for the flow to remain attached with the duct.

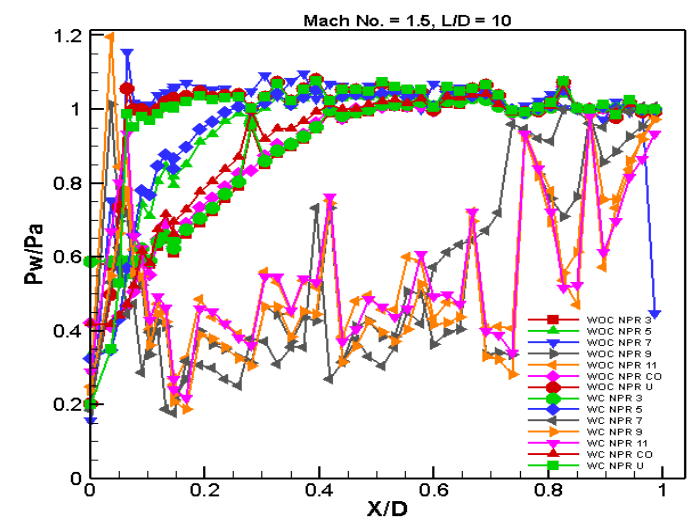

(a)

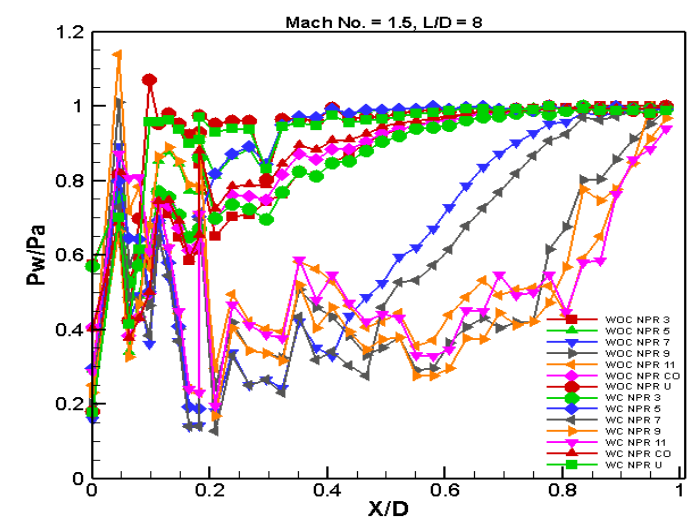

(b)

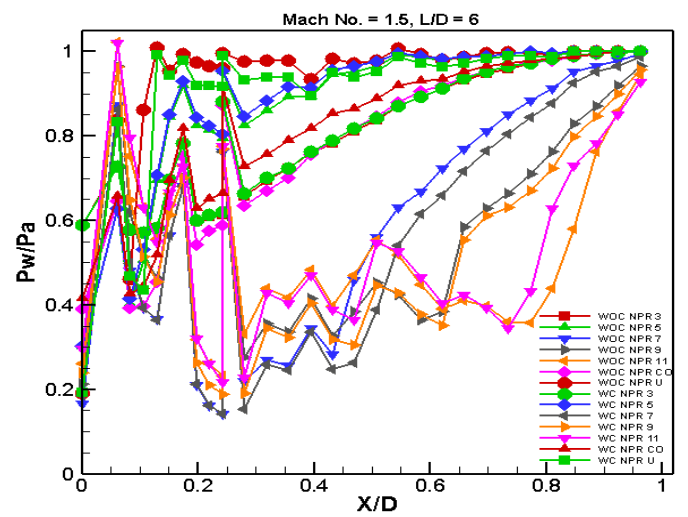

(c)

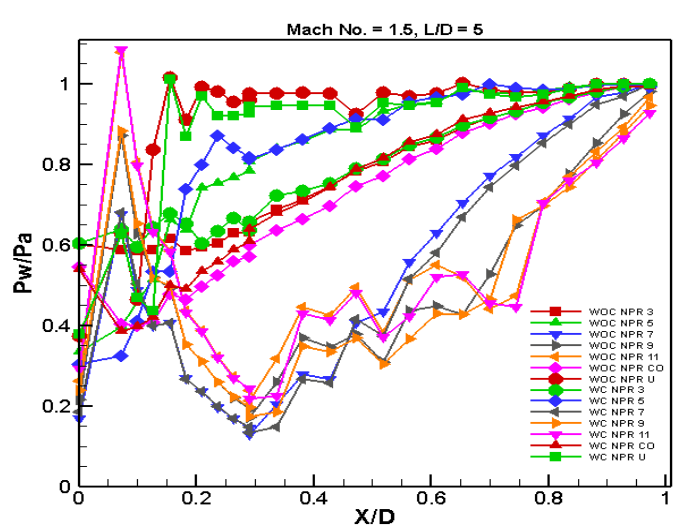

(d)

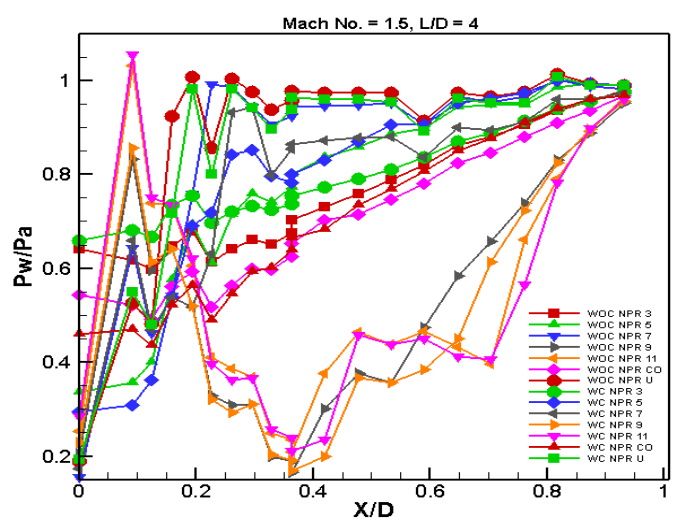

(e)

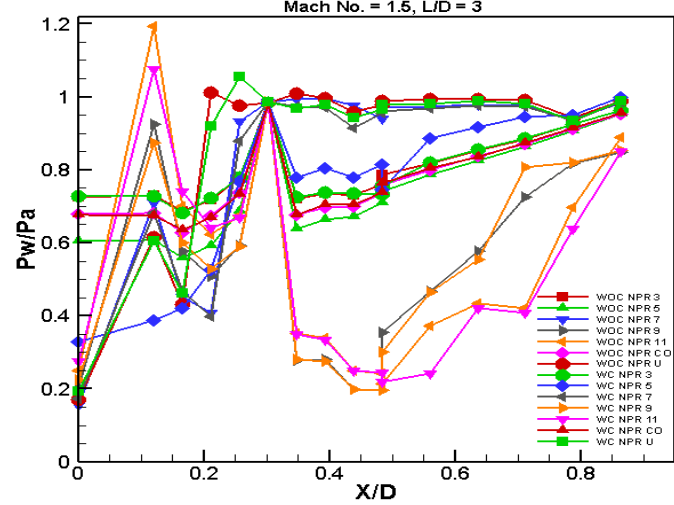

(f) 


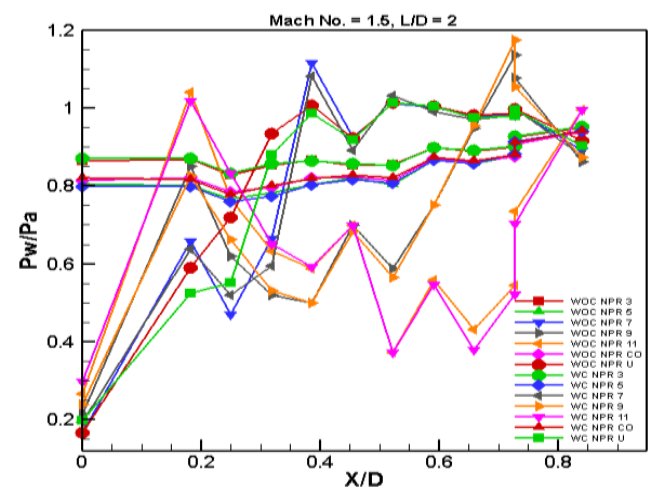

$(\mathrm{g})$

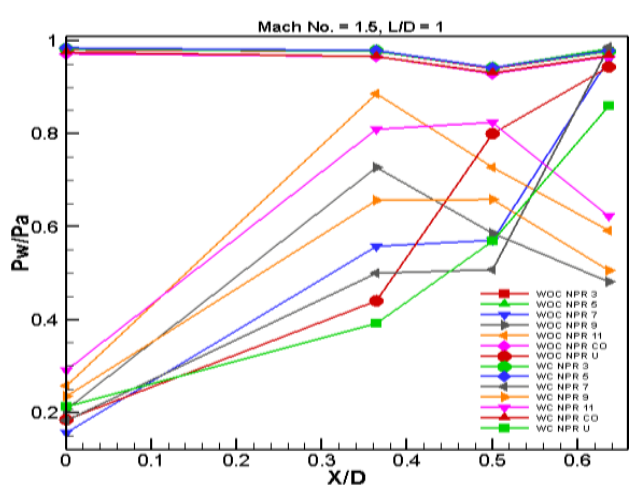

(h)

Figure 3. Wall pressure distribution

\section{CONCLUSION}

In present work, different level of expansion has been used to measure the wall pressure. Based on the above discussion we can conclude our results in the following manner:

- For all the higher level of expansion, the maximum increase of the wall pressure is one hundred twenty percent more than atmospheric is attained.

- The influence of the control mechanism on the wall pressure and the nature of the flow, the measured wall pressure remained identical with and without the deployment of the control.

- Microjets 4 in numbers are employed for the dynamic control of the base pressure and its effect on wall pressure focusing our attention, particularly on the duct flow nature.

- The value for Mach number and area ratio used during the experiment are 1.5 and 4.84 which corresponds to the Mach number which would lead to screech tone.

- The wavy nature of the flow is observed in view of the presence of the oblique shock wave or expansion wave.

- The minimum duct length required for the flow in the duct to remain attached seems to be $L / D=2$. Even though the flow in the duct seems to be attached even at $\mathrm{L} / \mathrm{D}=1$ for a higher level of expansion (i.e., NPR).

\section{ACKNOWLEDGMENT}

The corresponding author would like to thank and acknowledge International Islamic University Malaysia for the RIGS Grant (RIGS17-106-0681) for this research project.

\section{REFERENCES}

1. A. Saleel, M. A. A. Baig, and S. A. Khan, "Experimental Investigation of the Base Flow and Base Pressure of Sudden Expansion Nozzle," IOP Conf. Ser. Mater. Sci. Eng., vol. 370 no. $1,2018$.

2. S. A. Khan and E. Rathakrishnan, "Innovative Design, Analysis and Development Practices in Aerospace and Automotive Engineering (I-DAD 2018)," no. September 2019.

3. A. Krothapalli*, P. J. Strykowski, and C. J. King, "Origin of Streamwise Vortices in Supersonic Jets," AIAA J., vol. 36, no. 5, pp. 869-872, May 1998.

4. V. Kumar and F. Alvi, "Efficient Control of Separation Using Microjets," in 35th AIAA Fluid Dynamics Conference and Exhibit, American Institute of Aeronautics and Astronautics, 2005.

5. C. A. Hunter, "Experimental Investigation of Separated Nozzle Flows," J. Propuls. Power, vol. 20, no. 3, pp. 527532, 2004.

6. M. H. Tormalm, "Flow Control using Vortex Generators or Micro-Jets Applied in an UCAV Intake," in 52nd Aerospace Sciences Meeting, American Institute of Aeronautics and Astronautics, 2014.

7. F. F. Grinstein, "Vortex dynamics and entrainment in rectangular free jets," J. Fluid Mech., vol. 437, pp. 69-101, 2001

8. S. A. Khan, M. O. Ali, M. M. Riyadh, Z. Hossen, and N. M. Arefin, "Assessment of different turbulence models in simulating axisymmetric flow in suddenly expanded nozzles," Int. J. Eng. Technol., vol. 7, no. 3.29 Special Issue 29, pp. 39, 2018.

9. T. Gawehn, A. Gülhan, N. S. Al-Hasan, and G. H. Schnerr, "Experimental and numerical analysis of the structure of pseudo-shock systems in laval nozzles with parallel side walls," Shock Waves, vol. 20, no. 4, pp. 297-306, 2010.

10. S. A. Khan, A. Aabid, and A. S. C, "CFD Simulation with Analytical and Theoretical Validation of Different Flow Parameters for the Wedge at Supersonic Mach Number," no. March 2019.

11. M. Ahmed, A. Baig, and S. A. Khan, "A Study on Suddenly Expanded Flow at Different Levels of Over Expansion for Area Ratio 3.24," Int. J. Sci. Eng. Res., vol. 3, no. 8, 2012.

12. M. A. A. Baig, S. A. Khan, and M. Y. Khan, "Effect of Area Ratio on Base Pressure in a Suddenly Expanded Duct for Under Expanded Flow with Mach 1.87," Int. J. Mech. Ind. Eng., vol. 2, no. 1, pp. 2231-6477, 2012.

13. P. R. VISWANATH and S. R. PATIL, "Effectiveness of passive devices for axisymmetric base drag reduction at Mach 2," $J$. Spacecr. Rockets, vol. 27, no. 3, pp. 234-237, May 1990.

14. S. A. Khan and E. Rathakrishnan, "Control of suddenly expanded flow," Aircr. Eng. Aerosp. Technol. An Int. J., vol. 78, no. 4, pp. 293-309, 2006.

15. A. Krothapalli, E. Rajkuperan, F. Alvi, and L. Lourenco, "Flow field and noise characteristics of an impinging supersonic jet," J. Fluid Mech., vol. 392, pp. 155-181, 1999.

16. F. S. Alvi, C. Shih, R. Elavarasan, G. Garg, and A. Krothapalli, "Control of Supersonic Impinging Jet Flows Using Supersonic Microjets," AIAA J., vol. 41, no. 7, pp. 1347-1355, Jul. 2003.

17. S. A. Khan and E. Rathakrishnan, "Active Control of Suddenly Expanded Flows from Underexpanded Nozzles Part II," Int. J. Turbo Jet Engines, vol. 22, pp. 163-183, 2005.

18. S. A. Khan and E. Rathakrishnan, "Effect of Ribs on Suddenly Expanded Flows," AIAA J., vol. 39, no. 7, pp. 14021404, Jul. 2001

19. R. W. KRUISWYK and J. C. DUTTON, "Effects of a base cavity on subsonic near-wake flow," AIAA J., vol. 28, no. 11, pp. 1885-1893, Nov. 1990. 